\title{
Análisis de la Evolución de la Disponibilidad Léxica en la Interacción Pedagógica
}

\author{
Carolina Zambrano ${ }^{1 \star}$, Darío Rojas ${ }^{1}$, Pedro Salcedo ${ }^{1}$ y Jorge Valdivia ${ }^{1}$ \\ (1) Departamento de Metodología de Investigación e Informática Educacional, Facultad de Educación \\ Universidad de Concepción, Concepción, Chile. (e-mail: carolinazambrano@gmail.com; dfrojas@gmail.com; \\ psalcedo@udec.cl; jvaldivi@udec.cl)
}

${ }^{*}$ Autor a quien debe ser enviada la correspondencia

Recibido May. 24, 2018; Aceptado Jul. 31, 2018; Versión final Sep. 7, 2018, Publicado Feb. 2019

\begin{abstract}
Resumen
El objetivo del estudio es analizar la evolución de la disponibilidad léxica en la interacción pedagógica usando estadísticos descriptivos y una representación espacio vectorial. El diseño es longitudinal con medición pre y post. Así, se midió la evolución de la disponibilidad léxica de los estudiantes respecto al centro de interés, Estadística en un curso de Metodología de la Investigación. Participaron 62 estudiantes de dos carreras de pedagogía que generaron un total de 1747 vocablos. Los resultados revelaron que existe una diferencia estadísticamente significativa para la evolución del lexicón de los estudiantes, lo que concuerda con otros estudios similares. En conclusión, se sugiere la utilidad del enfoque propuesto como medida cuantitativa que permite verificar la evolución de la disponibilidad léxica de los estudiantes en la interacción pedagógica en un contexto específico.
\end{abstract}

Palabras clave: disponibilidad léxica; representación espacio vectorial; interacción pedagógica; educación superior; caudal léxico

\section{Analysis of the Evolution of the Lexical Availability in the Pedagogical Interaction}

\begin{abstract}
The objective of the study is to analyze the evolution of the lexical availability in the pedagogical interaction using descriptive statistics and a vectorial space representation. The design is longitudinal with pre and post measurement. Thus, the evolution of the lexical availability of the students with respect to the center of interest, Statistics in a course of Research Methodology, was measured. Sixty-two students from two pedagogy careers that generated a total of 1747 words participated in the study. The results revealed that there is a statistically significant difference for student lexicon evolution, which is consistent with other similar studies. In conclusion, it is suggested the usefulness of the proposed approach as a quantitative measure that allows verifying the evolution of the lexical availability of students in the pedagogical interaction in a specific context.
\end{abstract}




\section{INTRODUCCIÓN}

La interacción pedagógica es una acción fundamental del profesor que ayuda a promover dinámicas y/o estrategias didácticas que faciliten el aprendizaje de los estudiantes (Escobar, 2015; Heyd-Metzuyanim, 2013; Godoy-Ossa, Varas-Scheuch, Martínez-Videla, Treviño y Meyer, 2016). En este sentido, un estudio reciente en Chile ha reportado que "las interacciones pedagógicas participativas son relevantes para los aprendizajes y especialmente lo son para los jóvenes de menor rendimiento" (Agencia de Calidad de Educación, 2017, p.29). Asimismo, varios investigadores han evidenciado el impacto de la interacción pedagógica en el aula para el aprendizaje de los estudiantes (Escobar, 2015; Godoy-Ossa et al., 2016, Martinic et al., 2013; entre otros). Al contrario, una escasa interacción pedagógica en el aula afecta negativamente el aprendizaje de los estudiantes.

También, la interacción pedagógica hace posible que nuevos elementos del lenguaje, tanto propios como colectivos puedan ser adquiridos (Azkarai y Oliver, 2016; Escobar, 2015; Oliver y Mackey, 2003). En este ámbito, la interacción pedagógica se ha estudiado desde los procesos comunicativos derivados del uso del lenguaje y el intercambio verbal en el aprendizaje de una lengua, señalando que permite a cada participante internalizar el aprendizaje e incorporarlo a su propio aparato cognoscitivo (Oliver y Mackey, 2003; Oliver y Azkarai, 2017). En consecuencia, debido a que la interacción pedagógica se puede desarrollar a través de procesos de comunicación derivados del uso del lenguaje e intercambio verbal, es posible medir ese caudal léxico definiendo un contexto y tema de trabajo en el aula. Para medir el caudal léxico se puede usar la prueba de disponibilidad léxica que corresponde al análisis del subconjunto de palabras de mayor utilización en dicho proceso comunicativo (Valencia y Echeverría, 1999). Por ejemplo, si se define como contexto una asignatura de Metodología de Investigación y se propone medir la evolución del léxico disponible para el tema Estadística, el profesor deberá planificar y preparar actividades que permitan generar interacción en el aula con énfasis en el apoyo pedagógico (Pianta, Hamre y Mintz, 2012).

Respecto a estudios de análisis de disponibilidad léxica, cabe señalar que la mayoría de ellos se han desarrollado en España y Latinoamérica. La evidencia empírica se focaliza en investigaciones que han considerado como ámbito de estudio el léxico disponible en matemáticas (Cerda et al., 2017; Ferreira et al., 2014; Salcedo et al., 2013; Salcedo et al., 2017; Urzúa et al., 2006), disponibilidad léxica y comprensión lectora (Cepeda et al., 2017), estudios de léxico disponible de inglés como segunda lengua de instrucción formalizada (Germany y Cartes, 2000), en el ámbito de léxico disponible asociado a la categoría semántica de tecnología (Tome, 2016), léxico disponible de español como lengua extranjera (Serfati, 2016) y estudios de disponibilidad léxica asociados al proyecto panhispánico de léxico disponible que utiliza un estándar de 16 centros de interés tales como: los animales, el cuerpo humano y otros (Garzón y Penagos, 2016; Hernández-Muñoz, 2015; entre otros). Sin embargo, no existe una propuesta de análisis de la disponibilidad léxica en la interacción pedagógica que permita verificar la evolución de ésta usando una representación espacio vectorial.

En relación con la interacción pedagógica se debe señalar que la mayoría de los estudios en Chile son recientes y están situados en el contexto de la educación prebásica, básica y media (Godoy-Ossa et al., 2016; Leyva et al., 2015; Martinic et al., 2013; Martinic et al., 2015; entre otros). Estos estudios se han desarrollado gracias al proceso de evaluación docente en establecimientos públicos desde el año 2003. No obstante, en educación superior en Chile no existe igual interés en el tema. La interacción pedagógica se puede medir en tres dominios: apoyo emocional, organización de la sala y apoyo pedagógico (Pianta et al., 2012). En esta investigación se trabaja el dominio de apoyo pedagógico para la interacción pedagógica. Por ello, se generan acciones pedagógicas para su fomento en una asignatura de Metodología de Investigación con énfasis en el tema Estadística. El motivo de la elección de 'Estadística' como centro de interés está asociado a las orientaciones curriculares de los sujetos bajo estudio y a la orientación práctica que se le dio al curso para realizar una investigación exploratoria.

En suma, la investigación aborda las siguientes preguntas: ¿Existe una diferencia estadísticamente significativa entre la disponibilidad léxica de un grupo de estudiantes, representada a través del enfoque vectorial (Rojas, Zambrano y Salcedo, 2016, 2018), al principio y al final de una asignatura de Metodología de la Investigación respecto al centro de interés "estadística"? ¿el largo del lexicón mental de los estudiantes difiere significativamente entre el Pretest y Posttest de las carreras en estudio, respecto al centro de interés "estadística"? Para dar respuesta a estas preguntas de investigación, se estableció el siguiente objetivo: proponer un enfoque de medición de la disponibilidad léxica que permita verificar la evolución de elementos comunicativos en la interacción pedagógica, usando análisis estadísticos y una representación espacio vectorial.

\section{MÉTODO}

Se describe diseño, participantes, aplicación de la interacción pedagógica, proceso de análisis, entre otros. 


\section{Diseño}

La investigación tiene un diseño no experimental, longitudinal con medición pre y post.

\section{Participantes}

Participaron 62 estudiantes, 36 pertenecen a la carrera de Pedagogía en Inglés y 26 pertenecen a la carrera de Pedagogía en Matemática con edades entre 19 y 24 años $(M=22 ; S D=1.9)$. En ambas carreras el curso de Metodología de Investigación es obligatorio con contenidos de estadística aplicada a la investigación educativa. Cabe señalar que los estudiantes de Pedagogía en Ingles no tienen ningún curso de estadística ni antes, ni después del semestre en que cursan Metodología de Investigación. Por el contrario, los estudiantes de Pedagogía en Matemática si tienen curso de estadística un año después. Por ello, la elección de 'Estadística' como centro de interés está asociada a las orientaciones curriculares de los sujetos bajo estudio y a la orientación práctica que se le dio al curso para realizar una investigación exploratoria.

\section{Interacción Pedagógica en el Curso de Metodología de Investigación}

En este apartado se describen las actividades que se llevaron a cabo en el curso de Metodología de Investigación con el propósito de generar interacción pedagógica para el dominio de apoyo pedagógico que comprende cinco dimensiones: i) formato pedagógico, ii) comprensión del contenido, iii) análisis e indagación, iv) calidad de la retroalimentación y v) dialogo instruccional (Pianta et al., 2012). Todas estas dimensiones fueron cubiertas con las actividades que se describen a continuación.

Metodología de Investigación es una asignatura obligatoria en el currículum de las carreras de pedagogía que participan en la investigación y su propósito fue entregar herramientas de análisis para la elaboración de una investigación exploratoria en el ámbito educativo. Su carga horaria es de 4 horas académicas divididas en dos clases a la semana. Se distribuyó una clase para contenidos y una clase para el desarrollo y retroalimentación de la investigación. Las actividades para el dominio de apoyo pedagógico fueron diseñadas por la docente en la planificación didáctica que incluyó: que los estudiantes aplicaran una investigación exploratoria acotada a un semestre. Para este fin, se utilizó la metodología activa de proyectos Cattaneo (2017) que implicó que los estudiantes planificasen un proyecto de investigación en grupos de 3 a 4 personas y que posteriormente lo desarrollasen con el propósito de generar un ambiente de aprendizaje colaborativo entre los estudiantes y la docente (Willegems et al., 2017).

Para la planificación del proyecto de investigación la docente solicitó a cada equipo de investigación desarrollar una propuesta de investigación, entregar su documentación al inicio del semestre y defender oralmente su propuesta. Para monitorizar el desarrollo del proyecto se solicitó a cada equipo de investigación entregar una carta Gantt con las etapas y roles de cada integrante, y preparar tres exposiciones con el avance del proyecto donde cada exposición tenía el siguiente propósito: i) presentación del marco teórico e instrumentos de adquisición de datos, ii) exposición del avance de la investigación, iii) defensa final de investigación con sus resultados. También, una vez a la semana se desarrolló una reunión entre el equipo de estudiantes y la docente. En todas las etapas del proyecto los estudiantes recibían retroalimentación de la docente. Finalmente, para evaluar el proyecto se entregó la rúbrica de evaluación al inicio del semestre. En particular, para fomentar la interacción pedagógica para el dominio de apoyo pedagógico con énfasis en el tema estadística, se realizaron las siguientes actividades: uso del software libre PSPP para el análisis estadístico de datos, creación de apuntes con ejemplos específicos, promoción de la habilidad de análisis e indagación mediante el trabajo de los estudiantes en laboratorio de computación con software PSPP y retroalimentación continua.

\section{Instrumento}

El instrumento utilizado para la recolección de datos es el test de disponibilidad léxica (Valencia y Echeverría, 1999) que mide el léxico disponible que son las palabras que se presentan en la mente del estudiante de forma inmediata cuando se trata una categoría semántica también llamada centro de interés. El tiempo límite de respuesta fue de dos minutos para el centro de interés "estadística". Para el análisis se utilizan cinco índices tradicionalmente aplicados en los estudios de disponibilidad léxica: 1) Número de Lexicones (N): corresponde a la cantidad de individuos considerados en un grupo, por lo que también representa la cantidad de lexicones en un centro de interés; 2) Promedio de Respuestas (XR): indica cuántos vocablos en promedio ha respondido un grupo de individuos ante un estímulo o centro de interés específico; 3) Número de Palabras Diferentes (NPD): es un índice que da cuenta del total de vocablos que produce un grupo de sujetos para algún centro de interés, considerando cada palabra una única vez (a pesar de, posiblemente, haber sido indicada por más de un individuo); 4) Índice de Cohesión (IC): es un indicador del grado de coincidencia en las respuestas de los individuos. Este permite analizar comparativamente el léxico de un centro de interés en distintos grupos de sujetos, estableciendo cuan similares son las respuestas en cada uno de ellos. Se puede 
considerar como el grado de homogeneidad de un grupo respecto a su léxico disponible. El índice IC se obtiene del cociente entre el promedio de respuestas del grupo dividido por el total de palabras diferentes (XR/ NPD); 5) Índice de Disponibilidad Léxica (IDL): corresponde al grado de disponibilidad de un vocablo en el grupo, ante un estímulo o centro de interés. La forma más común de calcular este índice es utilizando la siguiente ecuación (1):

$$
i d l\left(v_{i}\right)=\frac{f_{1} \lambda^{0}+f_{2} \lambda^{1}+f_{3} \lambda^{2}+\cdots+f_{p} \lambda^{p-1}+\cdots+f_{t} \lambda^{t-1}}{N}
$$

En esta ecuación, $f_{p}$ es la frecuencia de aparición del vocablo $v_{i}$ en la posición $p$, siendo $p=0$ cuando el vocablo es indicado en primera posición y $p=t$ cuando el vocablo es señalado en la última posición $t$, con $t>0$. La expresión $\lambda^{p-1}$ es denominada tasa de sustitución o factor de ponderación a la posición (Salcedo et al., 2013), y su valor va decreciendo a medida que la posición es mayor. También, para el análisis se aplica el modelo espacio vectorial para los lexicones propuesto en Rojas et. al $(2016,2018)$. El modelo de espacio vectorial está basado en las matrices de frecuencia término-documento utilizadas comúnmente para representar palabras y documentos en los sistemas de recuperación de información (Salton et al. 1975; Chakrabarti, 2002).

\section{Procedimiento}

El test de disponibilidad léxica se aplicó al inicio del semestre y al final del semestre del periodo académico 2016-2. Los estudiantes respondieron de forma voluntaria y en papel el test de disponibilidad léxica para el centro de interés "estadística" en un tiempo de dos minutos.

\section{Proceso de Análisis de Datos}

En el proceso de análisis de datos se aplicaron dos enfoques. El enfoque tradicional usando los índices NPD, XR, IC e IDL. Y el enfoque propuesto por Rojas et. al $(2016,2018)$ que ocupa el modelo espacio vectorial para los lexicones empleando matrices de frecuencia término-documento, considerando de esta forma a cada lexicón como un documento y los vocablos como términos. Usar el modelo espacio vectorial representa un aporte en el ámbito de la investigación relacionada con la disponibilidad léxica porque incorpora en su formulación no sólo la frecuencia de palabras, sino que también un esquema de ponderación que permite considerar las particularidades de la información en su conjunto y su agrupación. De este modo, la comparación entre grupos se realizó mediante la representación vectorial de los lexicones (Rojas et al., 2016, 2018). Así, el lexicón de cada alumno es representado en una única dimensión como un valor escalado linealmente entre 1 y 7 . Luego, con los datos transformados en una única dimensión se utilizan pruebas estadísticas t-student para buscar diferencias significativas entre los grupos. O pruebas no paramétricas para datos no normales. Para los análisis se utiliza Matlab y SPSS. Las comparaciones entre el Pretest y Posttest se realizaron en el contexto de grupos independientes de estudiantes. Otro aspecto que se consideró es el preprocesamiento de las respuestas al test de disponibilidad léxica, por lo que los resultados y el procesamiento se realizaron sobre los vocablos lematizados y sin tildes. El proceso de lematización consiste en determinar los vocablos que pertenecen a un mismo paradigma flexivo o derivativo para convertirlos en una forma normal que representa a toda la clase (Kennedy, 2014).

\section{RESULTADOS}

El objetivo de esta sección es presentar los resultados de los dos enfoques de análisis aplicados.

Resultados por aplicación del enfoque estadístico de análisis del léxico

La Tabla 1 muestra la descripción de la muestra por grupo, se observa que se obtuvo en total 104 lexicones y 1747 vocablos.

Tabla 1. Descripción de la muestra, cantidad de lexicones por grupo.

\begin{tabular}{|l|l|l|l|l|}
\hline \multirow{2}{*}{ Grupo Curso } & Pretest & Posttest \\
\cline { 2 - 5 } & Lexicones & Vocablos & Lexicones & Vocablos \\
\hline Pedagogía en inglés & 16 & 208 & 36 & 664 \\
\hline Pedagogía en matemática & 26 & 402 & 26 & 473 \\
\hline
\end{tabular}

En la Tabla 2 se presentan los resultados del cálculo de los índices NPD, XR e IC para el centro de interés estadística y los grupos estudiados. Se observa que la cantidad de palabras distintas, NPD, aumenta entre el Pretest y Posttest para el caso de ambas carreras. Además, los índices de cohesión IC de Pedagogía en Matemática y Pedagogía en Inglés difieren, mostrando que el grado de coincidencia de las respuestas de los 
individuos aumenta para matemática y disminuye para inglés. Asimismo, se observa que el promedio en el largo de las respuestas XR, el número de palabras distintas NPD y la cantidad de palabras totales NPT es siempre mayor en el Posttest que en el Pretest. Otro resultado interesante es el alto valor de palabras distintas NPD producido por Pedagogía en Inglés en el Posttest, lo que tiene incidencia en el bajo índice de cohesión (IC) de este mismo grupo. Esto último sugiere que, a pesar de que los estudiantes de Pedagogía en Ingles en el Posttest producen las respuestas más largas y una cantidad mayor de palabras, la cohesión entre ellos, como medida de homogeneidad entre el léxico de los estudiantes, es relativamente bajo respecto al otro grupo. En este contexto se debe acotar que la diferencia en el tamaño muestral entre el Pretest y Posttest de Pedagogía en inglés produce un posible sesgo entre los índices NPD y NPT aunque no para XR e IC que se ajustan según la cantidad de individuos. La diferencia en el tamaño muestral se debe a que esos estudiantes no asistieron a clases el día que se aplicó la prueba de disponibilidad léxica y a que la aplicación fue voluntaria. En la Tabla 3, se muestra el índice IDL para los 10 vocablos más disponibles. Como se puede observar, los vocablos de Pedagogía en Matemática representan una relativa mayor similitud entre las dos pruebas. En el caso de Pedagogía en Inglés aparecen nuevos vocablos como ‘analisis' y 'varianza'.

Tabla 2. Índices de disponibilidad léxica grupales

\begin{tabular}{|l|c|c|c|c|c|}
\hline \multicolumn{1}{|c|}{ Grupo Curso y Test } & N & NPD & XR & IC & NPT \\
\hline Pedagogía en Ingles Pretest & 16 & 100 & 13.00 & 0,13 & 208 \\
\hline Pedagogía en Ingles Posttest & 36 & 231 & 18.44 & 0,0798 & 664 \\
\hline Pedagogía en Matemática Pretest & 26 & 161 & 16.08 & 0,0999 & 402 \\
\hline Pedagogía en Matemática Posttest & 26 & 171 & 18.19 & 0,1064 & 473 \\
\hline
\end{tabular}

Tabla 3. Índice de disponibilidad léxica de los vocablos por grupo y curso (vocablos lematizados y sin tilde)

\begin{tabular}{|l|l|l|l|l|l|l|l|}
\hline \multicolumn{2}{|c|}{ Ped. Ingles, Pre-Test } & \multicolumn{2}{c|}{ Ped. Inglés, Post-Test } & \multicolumn{2}{c|}{ Ped. Matemática Pre-Test } & \multicolumn{2}{c|}{ Ped. Matemática Post-Test } \\
\hline \multicolumn{1}{|c|}{ Vocablo } & IDL & \multicolumn{1}{c|}{ Vocablo } & IDL & \multicolumn{2}{c|}{ Vocablo } & IDL & \multicolumn{2}{c|}{ Vocablo } & IDL \\
\hline Numero & 0,6933 & Numero & 0,4745 & Moda & 0,4281 & Dato & 0,5674 \\
\hline Grafico & 0,5546 & Media & 0,3535 & Dato & 0,3771 & Muestra & 0,4128 \\
\hline Porcentaje & 0,5372 & Grafico & 0,3415 & Grafico & 0,3696 & Grafico & 0,3968 \\
\hline Matematica & 0,3458 & Varianza & 0,2650 & Media & 0,2932 & Media & 0,3846 \\
\hline Media & 0,2117 & Promedio & 0,2558 & Numero & 0,2920 & Variable & 0,3133 \\
\hline Población & 0,2114 & Dato & 0,2478 & Porcentaje & 0,2766 & Tabla & 0,2840 \\
\hline Dato & 0,1977 & Variable & 0,2366 & Muestra & 0,2606 & Moda & 0,2828 \\
\hline Resultado & 0,1821 & Muestra & 0,2288 & Promedio & 0,2502 & Varianza & 0,2791 \\
\hline Variable & 0,1763 & Hipotesis & 0,2223 & Variable & 0,2364 & Mediana & 0,2755 \\
\hline Promedio & 0,1636 & Analisis & 0,2220 & Frecuencia & 0,2193 & Analisis & 0,2231 \\
\hline
\end{tabular}

En los resultados de la Tabla 3, se observa que, para Pedagogía en Ingles Pretest, aparecen menos palabras técnicas sobre estadística. En este sentido, las palabras representan elementos más comunes como 'número', 'porcentaje', 'gráfico' y 'matemática'. En cambio, en Pedagogía en Ingles Post-Test, se observa una evolución de los vocablos más disponibles, incorporando elementos que hacen referencia a palabras más propias del lenguaje estadístico como ‘varianza', 'muestra' y ‘análisis'. Por otro lado, en Pedagogía en Matemática Pretest se observa una mayor presencia de vocablos relativos al lenguaje de la estadística, y aunque la diferencia con Pedagogía en Matemática Posttest no es tan evidente, los vocablos nuevos del Posttest muestran un léxico más técnico, incorporando palabras como 'varianza', 'media', 'hipotesis' y 'análisis'. En relación con lo anterior, es importante destacar que, tanto en Pedagogía en Matemática como en Pedagogía en Ingles, en el Posttest se presenta el vocablo 'análisis' que referencia a la utilidad que otorga la estadística para realizar análisis. En este sentido, el vocablo 'análisis' no se visibilizó en la disponibilidad léxica inicial de cada carrera.

La presencia del vocablo análisis en el Posttest de cada carrera sugiere que la modalidad de las clases, que propició el desarrollo de un proyecto, generó un espacio de interacción pedagógica basada en la dimensión apoyo pedagógico que posiblemente influyo en la aparición de este tipo de vocablos como parte del léxico disponible sobre la estadística. En concreto, el uso del software PSPP como herramienta de análisis de datos permitió involucrar al estudiante y comprender los conceptos claves en el proceso de análisis estadístico. Por ello, los estudiantes realizaron un proceso de análisis e indagación que fomenta habilidades de pensamiento de orden superior. Lo anterior se experimentó en el laboratorio de computación usando PSPP con la retroalimentación de la docente y un dialogo instruccional adecuado.

\section{Resultados por aplicación del modelo de espacio vectorial para el análisis del léxico}

Para confirmar los resultados estadísticos mostrados anteriormente, se construyó un modelo espacio vectorial basado en el enfoque (Rojas et al., 2016, 2018) utilizando una sola dimensión de representación y la función de peso global basada en el IDL de los vocablos. Por ello, el lexicón de cada alumno es representado como 
un solo valor, escalado entre 1 y 7 . Las medias de los lexicones escalados linealmente entre 1 y 7 para cada grupo (carrera) en pre-test y post-test usando enfoque espacio vectorial se presentan en la Fig. 1. Luego, se realizó un análisis comparativo de las medias de los lexicones escalados linealmente para cada carrera en el pre y post test.

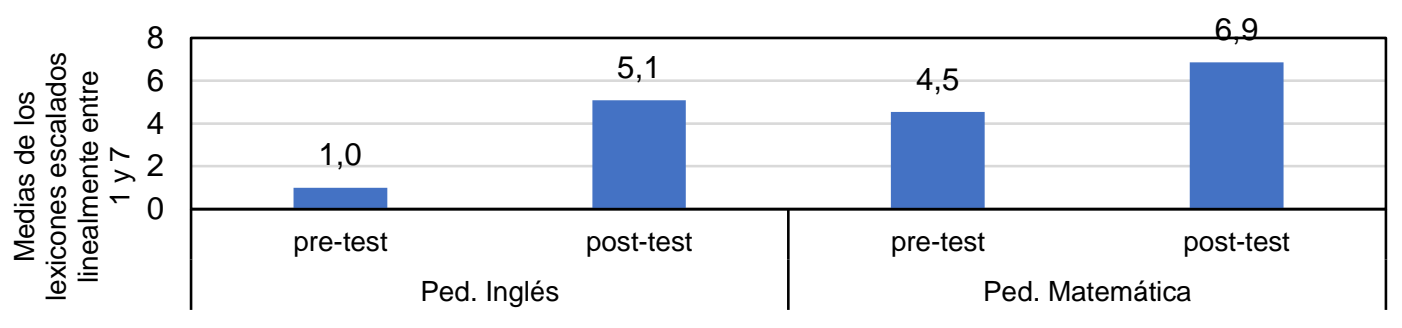

Resultados de cada carrera en pre test y post test

Fig. 1. Medias de los lexicones escalados linealmente entre 1 y 7 para cada grupo (carrera) en pre-test y post-test usando enfoque espacio vectorial (Rojas et al., 2016)

El análisis comparativo de las medias de los lexicones escalados para Pedagogía en Inglés mostró diferencias significativas entre los grupos Pretest y Posttest [t(52) $=-4.964, p<.001]$. En Pedagogía Matemática, también se observaron diferencias significativas entre los grupos Pretest y Posttest [t(52) $=-2.751, p=.008]$ y, por último, se obtuvo una diferencia significativa entre el Pretest de Pedagogía en Matemática y Pedagogía en Inglés [t(42) $=-4.271, p<.001]$, y entre el Posttest de los mismos cursos [ $\mathrm{t}(62)=-2.244, p=.029]$. Por otra parte, respecto al largo de las respuestas, se obtuvieron diferencias significativas entre el Pretest y Posttest de Pedagogía en Inglés [ $Z=-2.285, p<.022]$. No obstante, no se encontraron diferencias significativas entre el Pretest y Posttest, respecto al largo de las respuestas para Pedagogía en Matemática $[Z=-0.889, p=.374]$. En el mismo contexto, pero considerando la comparabilidad entre carreras, los resultados evidencian diferencias significativas respecto al largo de los lexicones del Pretest de Pedagogía en Matemática y Pedagogía en Inglés [ $Z=-2.269, p=.023]$. Sin embargo, no se obtuvo diferencias significativas entre los Posttest de estas mismas carreras $[Z=-3.329, p=.742]$.

Los resultados anteriores se pueden interpretar como una evolución significativa de los vocablos que componen el léxico sobre estadística, tanto en Pedagogía en Matemática como en Pedagogía en Inglés. No obstante, la cantidad de vocablos producidos durante el transcurso de la asignatura sólo fue significativamente diferente en Pedagogía Inglés. Esto último sugiere que la naturaleza de las carreras influye en el desarrollo y/o trasformación de la disponibilidad léxica de los estudiantes. Por esta razón, existe un efecto significativo en una carrera que no corresponde a la disciplina de la estadística como Pedagogía en Inglés. Por el contrario, en la carrera de Pedagogía en Matemática, el currículo está contextualizado en el ámbito numérico que incluye a la estadística.

\section{DISCUSIÓN}

En esta sección se presenta la discusión de los resultados de acuerdo con el objetivo de proponer un enfoque de medición de la disponibilidad léxica que permita verificar la evolución de elementos comunicativos en la interacción pedagógica, usando análisis estadísticos y una representación espacio vectorial. En este sentido, el enfoque propuesto representa una contribución a la enseñanza universitaria por cuanto puede ser aplicado en diferentes materias para medir la disponibilidad léxica en la interacción pedagógica. En consecuencia, el principal aporte del artículo es ofrecer un procedimiento para determinar cómo varía la disponibilidad léxica de los estudiantes al comienzo de un curso y al final de este.

Los hallazgos respecto a la pregunta, ¿existe una diferencia estadísticamente significativa entre la disponibilidad léxica de un grupo de estudiantes, representada a través del enfoque vectorial al principio y al final de una asignatura de Metodología de la Investigación, con respecto al centro de interés "estadística"? muestran que existe una diferencia significativa entre el léxico disponible al comienzo de la asignatura y al final para las dos carreras. Asimismo, existe una diferencia significativa entre carreras al comienzo de la asignatura que al final de la asignatura deja de ser significativa porque se homogeneiza el léxico disponible para ambas.

En relación a la pregunta, ¿El largo del lexicón mental de los estudiantes difiere significativamente entre el Pretest y Postest de las carreras de Pedagogía en Inglés y Pedagogía en Matemática con respecto al centro de interés "estadística"? Sólo se verificó parcialmente, debido a que el largo del lexicón mental de los estudiantes difiere significativamente sólo en pedagogía en Inglés, pero no para pedagogía en Matemática, aunque se debe acotar que esta comparación es sólo en base a la cantidad de vocablos producidos por los participantes y no para los vocablos mismo, los cuales si son diferentes significativamente según el método 
de espacio vectorial utilizado. Con esta evidencia empírica se corroboró la utilidad del enfoque propuesto como medida de evolución de la disponibilidad léxica de los estudiantes fomentada por la interacción pedagógica. También, los resultados obtenidos en esta investigación concuerdan con otros estudios que relacionan el índice NPD y la cantidad de años que un estudiante ha permanecido en una institución (Ferreira et al., 2014; Cepeda et al., 2017).

Para promover la interacción pedagógica respecto al dominio de apoyo pedagógico que se compone de cinco dimensiones (Pianta et al., 2012), se realizó una planificación didáctica que incluyó el desarrollo de un proyecto de investigación grupal (Cattaneo, 2017) que fue planificado, monitoreado y evaluado de forma sistemática con el propósito de generar espacios de aprendizaje, comunicación y colaboración. En este aspecto, esta investigación se relaciona con el planteamiento de Willegems et al. (2017) quienes en una revisión sistemática sobre colaboración entre profesores y estudiantes de pedagogía para la investigación educacional concluyen que se debe dar atención al papel de los formadores de profesores porque se ha identificado como tema clave en la formación de futuros profesores la experimentación de la interacción pedagógica y la colaboración.

En suma, la investigación presentada combina un dialogo interdisciplinario entre educación y ciencias de la computación porque liga aspectos de la interacción pedagógica con el uso del modelo espacio vectorial para representar los lexicones empleando matrices de frecuencia término-documento que luego se reducen a una dimensión para ser comparadas. De este modo, se hace posible la comparabilidad numérica entre los lexicones de dos grupos considerando en su representación a todos los vocablos y habilitando la aplicación de test estadísticos más allá de la comparación del largo promedio de palabras, sino que utilizando en su conjunto las representaciones de los lexicones en un espacio de vectores (Rojas et al., 2016; Rojas et al., 2018). Asimismo, la disponibilidad léxica puede ser utilizada como herramienta para la formación de grupos colaborativos en aula creando un método para este fin como el propuesto en (Rojas, Zambrano y Salcedo, en prensa). Las limitaciones del estudio están asociadas al tamaño de muestra y posibles sesgos asociados a las diferencias curriculares. Como proyección el diseño de la investigación puede ser mejorado incluyendo otro tipo de instrumentos para medir la interacción pedagógica.

\section{CONCLUSIONES}

1.Se ha propuesto un enfoque para medir la evolución de la disponibilidad léxica en la interacción pedagógica utilizando una representación espacio vectorial. Así, el lexicón de cada alumno es representado en un espacio vectorial y transformado en una única dimensión como un valor escalado linealmente entre 1 y 7 . Luego, con los datos transformados se aplican pruebas estadísticas para buscar diferencias significativas entre las medias de los grupos para corroborar empíricamente la evolución de la disponibilidad léxica.

2.El procedimiento para determinar cómo varía la disponibilidad léxica de los estudiantes al comienzo de un curso y al final de este, permite establecer diferencias cuantitativas considerando en su representación a todos los vocablos y puede ser aplicado a distintos contextos.

3.Las actividades creadas para el fomento de la interacción pedagógica en el curso de Metodología de Investigación con énfasis en el tema estadística pueden ser replicadas en carreras del área de la educación u otras.

\section{REFERENCIAS}

Agencia de Calidad de Educación, Interacciones pedagógicas participativas: el aporte de la retroalimentación efectiva y el diálogo igualitario en el aula, Chile, Ministerio de Educación (2017)

Azkarai, A. y R. Oliver, Negative feedback on task repetition: ESL vs. EFL child settings, doi:10.1080/09571736.2016.1196385, Language Learning Journal (2016)

Cattaneo, H., Telling Active Learning Pedagogies Apart: from Theory to Practice, doi: 10.7821/naer.2017.7.237, Journal of New Approaches in Educational Research, 6(1), 144-152 (2017)

Cepeda, M., A. Cárdenas y otros cinco autores, Relación entre disponibilidad léxica y comprensión lectora, en un contexto de educación técnico profesional rural, Revista Sophia Austral, ISSN: 0719-5605,1(1), 81-93 (2017)

Cerda, G., P. Salcedo, C. Pérez y V. Marín, Futuros Profesores de Matemáticas: Rol de la Disponibilidad Léxica, Esquemas de Razonamiento Formal en Logros Académicos Durante su Formación Inicial, doi:10.4067/S071850062017000100005, Formación universitaria, 10(1), 33-46 (2017)

Chakrabarti, S., Mining the Web Discovering Knowledge from Hypertext Data, 2ª Ed., 56-57, Morgan Kaufmann, USA (2002)

Escobar, M., Influencia de la Interacción Alumno-Docente en el Proceso Enseñanza-Aprendizaje, Paakat: Revista de Tecnología y Sociedad, 8 (2015)

Ferreira, A., P. Salcedo y M. Del Valle, Lexical Availability Study in the Field of Mathematics, Estudios Filologicos, (54), $69-84(2014)$ 
Garzón A. y L. Penagos, First Semester Undergraduate Students' Lexical Availability at a University in Villavicencio, Colombia, doi:10.15446/fyf.v29n2.60189, Forma y Función, 29(2), 63-84 (2016)

Godoy-Ossa, F., L. Varas-Scheuch y otros tres autores, Interacciones Pedagógicas y Percepción de los Estudiantes en Escuelas Chilenas que Mejoran: una Aproximación Exploratoria, doi:10.4067/S0718-07052016000400008, Estudios Pedagógicos (Valdivia), 42(3), 149-169 (2016)

Heyd-Metzuyanim, E., The Co-construction of Learning Difficulties in Mathematics Teacher-Student Interactions and their Role in the Development of a Disabled Mathematical Identity, doi:10.1007/s10649-012-9457-z, Educational Studies in Mathematics, 83(3), 341-368 (2013)

Hernández, R., C. Fernández y P. Baptista, Metodología de la Investigación, 6ª Ed., 136-137, McGraw-Hill, México (2014)

Hernández-Muñoz, N., The Assessment of Adult Lexical Competence: An Approach from Lexical Availability and Academic Specialization in Pre-university Students, Revista de Filología de la Universidad de La Laguna, 33, 79-99 (2015)

Germany, P. y N. Cartes, Léxico disponible en inglés como segunda lengua de instrucción formalizada, doi:10.4067/S0718-07052000000100003, Estudios Pedagógicos, 26, 39-50 (2000)

Kennedy, G., An Introduction to Corpus Linguistics, 2ª Ed., 206-209, Routledge, NY, USA (2014)

Leyva, D., C. Weiland y otros cinco autores, Teacher-Child Interactions in Chile and Their Associations with Prekindergarten Outcomes, doi:10.1111/cdev.12342, Child Development, 86(3), 781-799 (2015)

López, H., Los Estudios de Disponibilidad Léxica: Pasado y Presente, Boletín de Filología, 35, 245-259 (1993)

Martinic, S., C. Vergara y D. Huepe, Uso del Tiempo e Interacciones en la Sala de Clases. Un Estudio de Casos en Chile, doi:10.1590/S0103-73072013000100009, Pro-Posicoes, 24(1), 123-135 (2013)

Martinic, S. y M. Villalta, La Gestión del Tiempo en la Sala de Clases y los Rendimientos Escolares en Escuelas con Jornada Completa en Chile, doi:10.1016/j.pe.2013.03.001, Perfiles Educativos, 37(147), 28-49 (2015)

Michéa, R., Mots Fréquents et Mots Disponibles, un Aspect Nouveau de la Statistique du Langage, Langues Modernes, 47, 338-344 (1953)

Oliver, R. y A. Mackey, Interactional context and feedback in child ESL classrooms, doi:10.1111/1540-4781.00205, The Modern Language Journal, 87(4), 519-533 (2003)

Oliver, R. y A. Azkarai, Review of Child Second Language Acquisition (SLA): Examining Theories and Research, doi:10.1017/S0267190517000058, Annual Review of Applied Linguistics, 37, (62) (2017)

Pianta, R., B. Hamre y S. Mintz, Classroom Assessment Scoring System. Upper Elementary Manual, Charlottesville, Virginia, Teachstone (2012)

Rojas, D., C. Zambrano y P. Salcedo, A Proposal for Predicting Academic Achievement Throught a Vectorial Model of Lexical Availability, doi:10.21125/iceri.2016.1603, ICERI2016 Proceedings, 2799-2806 (2016)

Rojas, D., C. Zambrano y P. Salcedo, Metodología de Análisis de Disponibilidad Léxica en Estudiantes de Pedagogía a través de la Comparación Jerárquica de Lexicones, doi:10.4067/S0718-50062017000400002, Formación Universitaria, 10(4), 03-14 (2017)

Rojas, D., C. Zambrano y P. Salcedo, Un Enfoque de Reconocimiento de Patrones para el Análisis de Disponibilidad Léxica en Estudiantes de Pedagogía en Matemática, Estudios Filológicos, 61, 1-30 (2018)

Rojas, D., C. Zambrano y P. Salcedo, Método para la formación de grupos colaborativos mediante disponibilidad léxica, Revista Electrónica de Investigación Educativa, 21(3) (en prensa) 2018

Salton, G., A. Wong y C. Yang, A Vector Space Model for Automatic Indexing, Communications of the ACM, 18 (11), $613-$ 620 (1975)

Salcedo, P., A. Ferreira y F. Barrientos, Bayesian model for lexical availability of Chilean High School students in mathematics, doi:10.1007/978-3-642-38637-4_25, Lecture Notes in Computer Science, Springer Verlag, Natural and Artificial Models in Computation and Biology, 7930 (1), 245-253 (2013)

Salcedo, P., M. A. Pinninghoff, R. Contreras y J.F. Figueroa, An adaptive hypermedia model based on student's lexicon, doi:10.1111/exsy.12222, Expert Systems, 34(4) (2017)

Serfati, M., Lexical Availability of University-level Moroccan Students: General Results, doi: 10.20420/PhilCan.2016.105, Philologica Canariensia, 22, 105-116 (2016)

Tome, C., Vocabulary of Computing and Emerging Technologies, Characterization from Lexical Availability, CaracteresEstudios Culturales y Críticos de la Esfera Digital, 5(1), 112-138 (2016)

Urzúa, P., K. Sáez y M. Echeverria, Disponibilidad léxica matemática: análisis cuantitativo y cualitativo, doi:10.4067/S0718-48832006000200005, Revista de Lingüística Teórica y Aplicada, 44, 59-76 (2006)

Valencia, A. y M. Echeverría, Disponibilidad Léxica en Estudiantes Chilenos, Ediciones Universidad de Chile-Universidad de Concepción, Santiago, Chile (1999)

Willegems, V., E. Consuegra, K. Struyven y N. Engels, Teachers and pre-service teachers as partners in collaborative teacher research: A systematic literature review, Teaching and Teacher Education, 64, 230-245 (2017) 